\title{
PERFIL NUTRICIONAL DE TRABALHADORES DE UMA EMPRESA DE INFORMÁTICA DO SUL DO BRASIL
}

\author{
Carla Adriane Schneider ${ }^{1}$, Janaína Guimarães Venzke²
}

\begin{abstract}
Resumo: O presente estudo objetivou identificar o perfil nutricional de trabalhadores de uma empresa de informática no Rio Grande do Sul. Estudo de caráter transversal observacional, com 57 funcionários. Para determinar o perfil nutricional, realizou-se a aferição de peso, altura e circunferência abdominal, além da aplicação de um questionário de consumo alimentar, visando identificar aspectos relacionados à qualidade das refeições feitas por essa população. O nível de atividade física dos funcionários também foi avaliado, através da aplicação do questionário Baecke. Encontrou-se uma elevada prevalência de excesso de peso entre os funcionários, sendo que destes, 36,84\% apresentaram sobrepeso e 19,3\% obesidade. Em relação ao ganho de peso durante os anos de trabalho na empresa, a mediana foi de $5,75 \mathrm{~kg}$, sendo o percentil 25 de 0,425 kg e o percentil 75 de 9,025 kg. Observou-se que 57,9\% dos participantes estavam dentro dos valores considerados normais de circunferência abdominal. Pode-se observar prevalência de atividade física ocupacional sedentária. O excesso de peso encontrado entre este grupo de trabalhadores corrobora com o encontrado por outras pesquisas, reforçando a necessidade da criação de estratégias de promoção da saúde, visando melhor qualidade de vida.
\end{abstract}

Palavras-chave: Antropometria, estado nutricional, saúde do trabalhador, riscos ocupacionais.

\section{NUTRITIONAL PROFILE OF COMPUTER WORKERS IN SOUTHEARN BRAZIL}

\begin{abstract}
This present study aimed to identify the nutritional profile of computer workers in a company in Rio Grande do Sul. This was a cross-sectional observational study with 57 employees. To identify the nutritional profile, weight, height and abdominal circumference were measured and was applied a food consumption questionnaire to evaluate the quality of meals among workers. The physical activity level was also evaluated through the application of the Baecke questionnaire. There was a prevalence of excess weight among employees, with $36.84 \%$ being overweight and $19.3 \%$ obese. In relation to weight gain, the median was $5.75 \mathrm{~kg}$, the 25 th percentile being $0.425 \mathrm{~kg}$ and the 75 th percentile being $9.025 \mathrm{~kg}$. It was observed that $57.9 \%$ of the participants were within normal values of abdominal circumference. The study showed prevalence of sedentary occupational physical activity. The excess weight found among this group
\end{abstract}

1 Graduada em Nutrição pela Universidade Federal do Rio Grande do Sul. Residente no Programa Saúde da Família e Comunidade da Residência Multiprofissional em Saúde - GHC, Porto Alegre, RS.

2 Doutora em Biotecnologia pela Universidade Federal de Pelotas. Professora no Departamento de Nutrição da Universidade Federal do Rio Grande do Sul. 
of workers corroborates with that found by other researches, reinforcing the need to create strategies for health promotion, aiming a better quality of life.

Keywords: Anthropometry, nutritional status, occupational health, occupational risk.

\section{INTRODUÇÃO}

Nas últimas décadas, a população mundial vem passando por uma importante transição no seu estilo de vida, com reflexos nos padrões alimentares e, consequentemente, no perfil nutricional da população (WHO, 2003). Uma alimentação rica em gorduras, carboidratos refinados e açúcares, com baixo consumo de vegetais, grãos integrais e frutas, é característica do que se chama dieta ocidental (POPKIN; ADAIR; NG, 2012). Essas mudanças nos padrões alimentares e no estilo de vida, caracterizado como mais sedentário, estão associadas com o aumento do sobrepeso e obesidade na população mundial e, consequentemente, às comorbidades associadas ao excesso de peso (WHO, 2003). Diabetes, doenças cardiovasculares e alguns tipos de cânceres são algumas comorbidades que destacam-se na população e que comprometem a qualidade de vida dos indivíduos (APOVIAN, 2016; GUH et al., 2009).

Essas mudanças também são percebidas na população brasileira. Dados da POF 2008-2009 mostram um aumento significativo na prevalência da obesidade entre a população adulta, sendo de $12,4 \%$ entre os homens e $16,9 \%$ entre as mulheres. Além disso, a pesquisa mostra que mais de $50 \%$ da população encontra-se acima do peso (IBGE, 2010).

Aliado a essa mudança nos padrões alimentares, houve um importante crescimento da alimentação fora de casa em regiões urbanas no mundo todo (SANTOS et al., 2011). No Brasil, essa tendência também pode ser observada (BEZERRA et al., 2013). Com o crescimento das refeições fora do lar, pode-se perceber uma associação positiva entre esta prática e ganho de peso (BEZERRA; SICHIERI, 2009).

O ambiente de trabalho é considerado cenário sedentário para muitos trabalhadores. Nas últimas décadas, tem-se observado uma diminuição no número de trabalhadores que tenham atividades ocupacionais com um esforço físico moderado $(\mathrm{CHURCH}$, 2011). Conseguir um equilíbrio entre a ingestão energética e o gasto energético é um problema crescente entre essa população. A inatividade física no local de trabalho e as horas de trabalho, estariam relacionadas a uma diminuição do gasto energético, que associado a um abundante consumo de alimentos (aumento da ingestão energética) leva a um balanço energético positivo, resultando em excesso de peso. $\mathrm{O}$ excesso de massa corporal entre trabalhadores foi associado com maior absenteísmo e maiores gastos com saúde por parte das empresas, estando também associado a riscos ocupacionais, como ferimentos, estresse, asma e desordens musculoesqueléticas (SCHULTE et al., 2007).

Como a maioria dos adultos passa um terço do seu dia no trabalho, esse ambiente estaria relacionado com boas oportunidades para tentar influenciar o comportamento 
dos funcionários, sendo alvo de promoção da saúde de muitos programas. Exercício e comportamento alimentar dos empregados são determinados não somente por escolhas conscientes, mas também pelos processos inconscientes, ou hábitos. Assim, informação através de educação alimentar e nutricional ou incentivo à redução da inatividade física, seriam influentes na hora de promover um comportamento mais saudável entre os trabalhadores (ANDERSON et al., 2009). Um estudo com trabalhadores do estado de Missouri, nos Estados Unidos, demonstrou que as relações sociais e a sensação de que a empresa se preocupa com a saúde dos seus funcionários, contribui para que os trabalhadores tenham hábitos alimentares mais saudáveis e pratiquem alguma atividade física (TABAK et al., 2015).

É fundamental conhecer sua população alvo para que se possam elaborar estratégias eficazes de promoção da saúde e isso inclui as ações de nutrição. Sendo assim, conhecer o perfil nutricional dos indivíduos se faz necessário. Considerando-se os aspectos abordados sobre os padrões alimentares, aumento da prevalência do excesso de peso na população em geral e as particularidades dos ambientes de trabalho, o objetivo deste estudo foi avaliar o perfil nutricional de um grupo de trabalhadores, a fim de contribuir com programas de vigilância em saúde do trabalhador.

\section{MATERIAL E MÉTODOS}

A presente pesquisa baseia-se num estudo observacional transversal, realizado em trabalhadores de uma empresa de informática da região metropolitana de Porto Alegre, Rio Grande do Sul, Brasil. A empresa foi escolhida por conveniência, por conhecimento da chefia, que autorizou a coleta de dados. O local conta com 138 colaboradores. Todos foram convidados a participar voluntariamente do estudo, sendo explicado a eles o objetivo do mesmo e seus procedimentos.

A coleta dos dados antropométricos, de perfil alimentar e de atividade física ocorreu no mês de junho de 2016, na própria empresa. A avaliação antropométrica foi realizada através da medida de peso, estatura e circunferência abdominal. A massa corporal foi aferida uma vez, em quilogramas $(\mathrm{kg})$, utilizando balança portátil eletrônica da marca Welmy, modelo W-200 M. Para a medida, todos os trabalhadores estavam descalços, com os bolsos vazios e vestindo suas vestes de trabalho, calça jeans e camisa de manga curta. A estatura foi aferida uma vez, em centímetros, com estadiômetro portátil da marca Alturexata com extensão de $200 \mathrm{~cm}$ e variação de $1 \mathrm{~mm}$. Os indivíduos estavam descalços, em posição ortostática e com os olhos no plano de Frankfört. A circunferência abdominal foi medida utilizando fita métrica, no ponto médio entre a última costela e a crista ilíaca.

Foi solicitado aos participantes, no momento da coleta de dados, que eles referissem o seu peso no início das atividades laborais na empresa. Também, eles foram questionados em relação ao tempo de trabalho no local. 
Com o peso e a altura foi calculado o índice de massa corporal (IMC) e a classificação foi realizada de acordo com o preconizado pela Organização Mundial da Saúde (WHO, 1995). Foram considerados eutróficos os indivíduos que apresentaram IMC entre 18,50 e $24,99 \mathrm{~kg} / \mathrm{m}^{2}$, sobrepeso entre 25 a $29,99 \mathrm{~kg} / \mathrm{m}^{2}$ e obesidade IMC superior a $30 \mathrm{~kg} / \mathrm{m}^{2}$. A circunferência abdominal foi utilizada para avaliar risco cardiovascular, sendo considerado de risco e alto risco, respectivamente, os valores de circunferência abdominal entre 94 e $102 \mathrm{~cm}$ e $>102 \mathrm{~cm}$ para homens e entre 80 e $88 \mathrm{~cm}$ e $>88 \mathrm{~cm}$ para mulheres.

Os funcionários responderam a um questionário, onde as questões iniciais objetivavam conhecer melhor onde esses indivíduos realizam mais frequentemente $o$ almoço e quais os fatores que eles consideram na hora de escolher onde irão realizar suas refeições. Além disso, buscou-se através de questões conhecer a percepção deles em relação a ganho ou perda de peso após iniciarem suas atividades laborais na empresa. O restante do questionário continha questões relacionadas ao consumo alimentar. Estas foram adaptadas do teste "como está sua alimentação?" do Guia Alimentar para a População Brasileira de 2006, com o objetivo de se ter uma ideia geral de como é o consumo de frutas, verduras, açúcares e gorduras pelos funcionários.

Utilizou-se o questionário Baecke para investigar o nível de atividade física dos indivíduos, sendo este dividido em atividade física ocupacional, exercício físico no lazer, atividade física de lazer e de locomoção (BAECKE; BUREMA; FRIJTERS, 1982).

Para apresentação da caracterização da amostra utilizou-se para as variáveis categóricas, percentual e número absoluto de indivíduos. Para as variáveis quantitativas, utilizou-se média e desvio padrão, e, quando apropriado, mediana e intervalo interquartil. Para verificar a associação das variáveis categóricas utilizou-se o teste quiquadrado. As análises estatísticas foram realizadas no programa Statistical Package for the Social Sciences (SPSS) versão 18.0, e os resultados foram considerados significativos quando $\mathrm{p} \leq 0,05$.

O presente estudo foi submetido ao Comitê de Ética em Pesquisa com Seres Humanos da Universidade Federal do Rio Grande do Sul (UFRGS), com o número de parecer 1.670.102 e segue a Resolução no 466/2012 de 12 de dezembro de 2012, que regulamenta a pesquisa com seres humanos (BRASIL, 2013). Todos os participantes assinaram o Termo de Consentimento Livre e Esclarecido de acordo com as normas estabelecidas pela Resolução 196/96 do Conselho Nacional de Saúde.

\section{RESULTADOS E DISCUSSÃO}

Dos 138 trabalhadores da empresa, 57 aceitaram participar do estudo, sendo 15 do sexo feminino e 42 do sexo masculino. Por se tratar de uma empresa com maioria de funcionários homens, o sexo masculino foi prevalente na pesquisa. A média de idade foi de 28,88 anos, com mínima de 18 e máxima de 48 anos. Analisando o tempo de 
trabalho no local, pode-se observar uma média de 5,13 anos, variando de 3 meses a 17 anos.

Em relação ao perfil antropométrico descrito na Tabela 1, pode ser observado um excesso de peso em 56,14\% $(\mathrm{n}=32)$ dos funcionários. Em relação à circunferência abdominal, mais da metade dos participantes estavam dentro dos valores considerados normais. Uma grande parcela dos funcionários, 75,4\% $(\mathrm{n}=43)$, afirmam terem mudado de peso após o início das atividades laborais na empresa, sendo que destes, 64,9\% ( $\mathrm{n}=37$ ) ganharam peso ao longo dos anos. A mediana do ganho de peso foi de $5,75 \mathrm{~kg}$, sendo o percentil 25 de $0,425 \mathrm{~kg}$ e o percentil 75 de $9,025 \mathrm{~kg}$.

Tabela 1 - Perfil antropométrico dos funcionários

\begin{tabular}{|c|c|c|c|c|}
\hline Características & $\begin{array}{l}\text { Homens } \\
\text { n (\%) }\end{array}$ & $\begin{array}{l}\text { Mulheres } \\
\text { n (\%) }\end{array}$ & $\mathrm{p}$ & $\begin{array}{c}\text { Amostra Total } \\
\text { n (\%) }\end{array}$ \\
\hline \multicolumn{5}{|l|}{ IMC } \\
\hline Eutrofia & $18(42,9)$ & $7(46,7)$ & \multirow{3}{*}{$0,947^{*}$} & $25(43,9)$ \\
\hline Sobrepeso & $16(38,1)$ & $5(33,3)$ & & $21(36,8)$ \\
\hline Obesidade & $8(19,0)$ & $3(20,0)$ & & $11(19,3)$ \\
\hline \multicolumn{5}{|l|}{$\mathrm{CA}(\mathrm{cm})$} \\
\hline Normal & $26(61,9)$ & $7(46,7)$ & \multirow{3}{*}{$0,591^{*}$} & $33(57,9)$ \\
\hline Risco & $8(19,0)$ & $4(26,7)$ & & $12(21,1)$ \\
\hline Risco Aumentado & $8(19,0)$ & $4(26,7)$ & & $12(21,1)$ \\
\hline
\end{tabular}

Fonte: Construção da autora.

Notas: *Teste qui-quadrado de Pearson.

Buscou-se investigar onde os trabalhadores realizam mais frequentemente suas refeições, uma vez que eles recebem vale-refeição. Mais da metade $(n=30)$ dos participantes almoçam em casa ou levam almoço para a empresa, sendo que 45,6\% $(\mathrm{n}=26)$ almoçam em restaurante. Um indivíduo referiu que substitui o almoço por lanches. Já em relação aos fatores que influenciam na hora de escolher onde irão realizar suas refeições, 31,6\% ( $\mathrm{n}=18)$ dos trabalhadores consideraram a comodidade como mais importante; $29,8 \%(\mathrm{n}=17)$ o sabor; $19,3 \%(\mathrm{n}=11)$ o custo; $19,3 \%(\mathrm{n}=11)$ a saúde/valor nutritivo.

Pode-se observar um perfil alimentar bem variado entre os trabalhadores participantes da pesquisa. Em relação à frequência de consumo dos alimentos, que foram divididos em grupos, notam-se resultados diferenciados, como mostra a tabela 2.

Em relação ao consumo de carnes, $78,9 \%(\mathrm{n}=45)$ dos participantes consome carnes de qualquer tipo todos os dias; $8,8 \%(n=5)$ menos de 2 a 3 vezes na semana; $10,5 \%$ $(\mathrm{n}=6)$ de 4 a 5 vezes na semana. Apenas 1 participante não consome carnes. Sobre a ingestão de leite e seus derivados, chama a atenção que somente $35,1 \%(n=20)$ dos 
trabalhadores costuma consumi-los diariamente. $31,5 \%(\mathrm{n}=18)$ consome menos de 2 a 3 vezes na semana e 31,6\% ( $n=18)$ consome de 4 a 5 vezes na semana. Apenas 1 participante não consome leite e seus derivados. Dos 57 participantes, 29 (50,9\%) consome leite e derivados integrais e $27(47,4 \%)$ com baixo teor de gorduras.

Tabela 2 - Frequência semanal de consumo dos diferentes grupos de alimentos

\begin{tabular}{|c|c|c|c|c|}
\hline Grupo de alimentos & $\begin{array}{c}\text { Raramente ou } \\
\text { nunca (\%) }\end{array}$ & $\begin{array}{c}\text { Todos os dias } \\
\text { (\%) }\end{array}$ & $\begin{array}{c}<2-3 x \text { semana } \\
(\%)\end{array}$ & $\begin{array}{c}4-5 \times \text { semana } \\
(\%)\end{array}$ \\
\hline Frutas & 19,3 & 28,1 & 26,3 & 26,3 \\
\hline Legumes e verduras & 3,5 & 35,1 & 35,1 & 26,3 \\
\hline Leguminosas & 10,5 & 26,3 & 29,9 & 33,3 \\
\hline Cereais & 1,8 & 56,1 & 8,8 & 33,3 \\
\hline Pães & 3,5 & 47,4 & 26,4 & 22,8 \\
\hline Frituras e embutidos & 14,0 & 8,8 & 59,7 & 17,5 \\
\hline $\begin{array}{l}\text { Doces, refrigerantes e } \\
\text { sucos industrializados }\end{array}$ & 15,8 & 10,5 & 52,7 & 21,1 \\
\hline
\end{tabular}

Fonte: Construção da autora

Em relação à prática de atividade física, $82,5 \%(\mathrm{n}=47)$ dos participantes afirmaram terem praticado pelo menos um tipo de atividade física nos últimos 12 meses. Destes, $17 \%(n=8)$ praticaram entre 120 e 180 minutos semanais, enquanto que $36,2 \%(n=17)$ praticaram mais de 180 minutos semanais. Sobre a atividade física ocupacional, 52 participantes afirmam ficarem sempre sentados durante o trabalho e o restante $(n=5)$ quase sempre sentados. Andar durante o expediente mostrou-se ser um hábito raro para $94,7 \%(n=54)$ dos participantes.

A hipótese de que seria encontrada uma prevalência elevada de excesso de peso no grupo de trabalhadores estudado por esta pesquisa foi confirmada através dos dados antropométricos coletados. Esse excesso de peso $\left(\mathrm{IMC}>25 \mathrm{~kg} / \mathrm{m}^{2}\right)$ observado está de acordo com o que outras pesquisas realizadas no Brasil encontram e reflete o crescente quadro de sobrepeso e obesidade da nossa população. Em estudo realizado com 105 trabalhadores de uma empresa de tecnologia do estado de São Paulo, foi encontrada prevalência de $54,3 \%$ de excesso de peso na população. Essa é uma prevalência similar à encontrada no presente estudo e com um grupo de trabalhadores do mesmo ramo, com uma atividade laboral sedentária (FAGGION; MONTEIRO, 2009). Em pesquisa realizada com funcionários de uma UAN (Unidade de Alimentação e Nutrição), realizada no Serviço de Nutrição e Dietética do Hospital de Clínicas de Porto Alegre, a prevalência de excesso de peso observada entre os trabalhadores foi de 60,8\% (SIMON et al., 2014). No estudo de Castro et al. (2004), realizado com 65 trabalhadores de uma 
empresa metalúrgica no Rio de Janeiro, foi encontrado excesso de peso em 44,6\% dos funcionários.

Considerando-se estudos com uma amostra maior que a da presente pesquisa, como o de Fernandes e Vaz (2012), com 366 trabalhadores de uma empresa de construção civil, grande parte da amostra encontrou-se com sobrepeso e obesidade (145 dos funcionários, ou seja, 39,6\%). Outro estudo, realizado com 364 trabalhadores, encontrou 70,9\% de excesso de peso entre os participantes (STOLTE, et al., 2006). No estudo de Hofelmann e Blank (2009), com 475 trabalhadores, onde a maioria da amostra também era constituída por homens, a prevalência de excesso de peso encontrada foi de $53 \%$ e a maioria dos trabalhadores não apresentaram obesidade abdominal, assim como no presente estudo.

Assim, pode-se perceber que mesmo em estudos com trabalhadores de diferentes áreas e com diferentes níveis de atividade física ocupacional, o excesso de peso é prevalente.

Em relação à alteração de peso, a mínima encontrada foi de $-15,05 \mathrm{~kg}$ e a máxima de $24,45 \mathrm{~kg}$. Por isso, utilizou-se a mediana dos dados para expressar o ganho de peso entre os funcionários, uma vez que a média foi de 5,29 \pm 7,59 kg.

É sabido que o excesso de peso está relacionado com diversas doenças crônicas não transmissíveis, como diabetes e doenças cardiovasculares (ABESO, 2016). Em trabalhadores, a obesidade pode estar associada a maior absenteísmo, mais gastos com saúde, além de menor produtividade (SCHULTE et al., 2007). Para avaliar o risco de complicações metabólicas associadas à obesidade em homens e mulheres, utiliza-se a circunferência abdominal, onde uma circunferência $>94 \mathrm{~cm}$ nos homens e $>80 \mathrm{~cm}$ nas mulheres é identificada como marcadora de risco, e os valores $>102 \mathrm{~cm}$ nos homens e $>88 \mathrm{~cm}$ nas mulheres sinalizam alto risco para o desenvolvimento de comorbidades. Dentre os indivíduos estudados, $42,2 \%(\mathrm{n}=24)$ apresentaram a medida da circunferência abdominal alterada, diferente do encontrado entre trabalhadores de uma UAN, onde $77,3 \%$ dos participantes apresentaram CA alterada. Por outro lado, Castro et al. (2004), analisando apenas trabalhadores do sexo masculino de uma empresa metalúrgica, observaram que somente $25 \%$ estavam com valores de circunferência abdominal acima de $94 \mathrm{~cm}$. Entre trabalhadores de uma empresa de tecnologia da informação de São Paulo, 47,4\% dos participantes apresentaram medida de CA alterada, prevalência similar à encontrada pelo presente estudo (FAGGION; MONTEIRO, 2009). As diferenças entre resultados podem se dar devido ao tipo de atividade laboral exercida pelos diferentes trabalhadores avaliados.

A Organização Mundial da Saúde preconiza que a ingestão diária de frutas e hortaliças seja de pelo menos 400 gramas, equivalendo, aproximadamente, ao consumo diário de cinco porções desses grupos de alimentos. Cerca de $60 \%$ das mortes em todo o mundo e de $45 \%$ da morbidade global estão relacionadas às doenças crônicas não transmissíveis (WHO, 2003). De acordo com a OMS, o consumo diário de frutas e 
verduras estaria relacionado à prevenção de doenças como, cardiopatias, diabetes tipo 2, obesidade e alguns tipos de cânceres.

O Vigitel - Vigilância de Fatores de Risco e Proteção para Doenças Crônicas por Inquérito Telefônico, em sua última publicação de resultados sobre a população brasileira, considerou como regular um consumo de frutas e hortaliças mais do que 5 vezes na semana. Em Porto Alegre, $42 \%$ dos participantes da pesquisa se enquadraram nesse grupo de pessoas (VIGITEL, 2017).

O presente estudo encontrou que $26,3 \%$ dos trabalhadores consomem frutas de 4 a 5 vezes na semana e que $28,1 \%$ consome todos os dias, ou seja, mais da metade $(\mathrm{n}=31)$ dos participantes possui um consumo de frutas próximo do recomendado. Já em relação ao consumo de verduras, $26,3 \%$ dos indivíduos consome de 4 a 5 vezes na semana e $35,1 \%$ consome todos os dias, ou seja, $61,4 \%(n=35)$ dos trabalhadores possui um consumo de verduras e hortaliças próximo do recomendado. Os dados encontrados são semelhantes e até superiores aos encontrados para a população de Porto Alegre. Por outro lado, em estudo realizado no Paraná com trabalhadores de uma empresa de fornecimento de energia, os autores encontraram um consumo diário bem inferior de frutas, 6,11\%, e de hortaliças, 11,36\% (SEBASTIÃO et al., 2015).

Verificou-se que o consumo de leguminosas é frequente para a maioria dos participantes deste estudo, sendo que 59,6\% ( $n=39)$ dos indivíduos inclui alguma leguminosa nas suas refeições pelo menos 4 vezes na semana. Dessa forma, podese perceber que as leguminosas são alimentos tradicionais na alimentação destes trabalhadores. Em estudo realizado por Souza et al. (2013), foi demonstrado que o feijão é a principal leguminosa consumida no país e faz parte da dieta básica do brasileiro. $\mathrm{O}$ VIGITEL encontrou que a frequência de consumo de feijão cinco ou mais vezes na semana, considerando todo o país, é de $61,3 \%$. Em Porto Alegre, observa-se que essa frequência é de 45,5\% (VIGITEL, 2017). Porém, em estudo realizado por Sebastião et al. (2015), também com trabalhadores, os autores encontraram que apenas 5,67\% dos participantes consomem algum tipo de leguminosa diariamente. Diferente do presente estudo, onde o consumo diário encontrado foi de $26,3 \%$.

Segundo Sarno et al. (2013), o consumo de sódio da população brasileira excede em mais de duas vezes o consumo máximo recomendado pela Organização Mundial da Saúde, que é de $2 \mathrm{~g} /$ dia. A forma de consumo mais tradicional do sódio é através do sal de cozinha e de condimentos à base de sal. Entretanto, os alimentos processados e ultraprocessados estão passando a contribuir cada vez mais para este alto consumo, por se fazerem cada vez mais presentes na alimentação da população brasileira. No Brasil, pode-se observar um aumento na relação de refeições fora de casa, de $24 \%$ para $31 \%$, entre 2002/03 e 2008/09 (IBGE, 2010).

Em estudo realizado em Goiânia que avaliou os teores de sal e gordura nas preparações de restaurantes comerciais da cidade, pode-se observar também que realizar refeições em restaurantes está associado ao maior consumo de sódio e gordura (KUNERT et al., 2013). Além disso, estudos demonstram uma associação positiva 
entre fazer refeições fora de casa com o aumento do IMC e rápido ganho de peso (BEZERRA; SICHIERI, 2009; DUFFEY et al., 2007). Dentre os participantes do presente estudo, um pouco menos da metade $(n=26)$ almoçam em restaurante. Juntamente com a importante prevalência de excesso de peso encontrada entre os indivíduos e a atividade laboral baixa, pode-se observar que esta população está exposta a fatores de risco para diversas comorbidades, como diabetes, hipertensão e doenças cardiovasculares.

Sobre a atividade física ocupacional dos funcionários, o estudo demonstra que a maioria dos entrevistados passa a maior parte do tempo do trabalho em frente ao computador $(91,2 \%)$. Quanto às atividades físicas de lazer, observa-se que uma grande parcela dos participantes realizou pelo menos um tipo de atividade física em seus momentos de lazer nos últimos 12 meses. A recomendação que se tem para atividade física no lazer é de, pelo menos, 150 minutos semanais de atividade física de intensidade leve ou moderada ou de, pelo menos, 75 minutos de atividade física de intensidade vigorosa (IBGE, 2014). No entanto, no grupo estudado, considerando-se a totalidade dos participantes, mais da metade $(n=32)$ não atinge a recomendação de realizar pelo menos 150 minutos de atividade física semanal.

As evidências mostram que a atividade física regular é protetora para o ganho de peso excessivo. Por outro lado, hábitos sedentários, em especial ocupações sedentárias, favorecem o excesso no ganho de peso (BARRETO et al., 2005). No Brasil, de acordo com a Pesquisa Nacional de Saúde, 27,1\% dos homens com 18 anos ou mais praticavam o nível recomendado de atividade física no lazer, enquanto que entre as mulheres este percentual foi mais baixo, de 18,4\%. A média brasileira foi de 22,5\% (IBGE, 2014).

\section{CONSIDERAÇÕES FINAIS}

Considerando-se o crescimento das atividades laborais consideradas mais sedentárias e o aumento da prevalência de padrões alimentares considerados não saudáveis, o presente estudo avaliou o perfil nutricional e características da alimentação de trabalhadores de uma empresa de informática.

No presente estudo, a prevalência de excesso de peso e o ganho de peso entre os trabalhadores após iniciarem as atividades laborais na empresa, corroboram com o encontrado em outras pesquisas, reforçando a necessidade de se dar mais atenção para a alimentação e saúde dos trabalhadores em geral.

Sabe-se que o desequilíbrio entre consumo e gasto energéticos estão associados a ganho de peso e, consequentemente, diversas doenças crônicas não transmissíveis. Dessa forma, é fundamental que se trabalhe a promoção da saúde nos mais diversos grupos populacionais. Como um adulto passa boa parte do seu dia em sua atividade laboral, uma estratégia seria promover atividades de educação nutricional no mesmo. 
Dessa forma, como perspectivas futuras para o presente trabalho, vê-se necessário trabalhar com os colaboradores da empresa onde foi realizada a pesquisa, educação alimentar e nutricional. Além disso, atividades de promoção da saúde em geral, estimulando a prática de atividade física. São estratégias de mudança de estilo de vida fundamentais para garantir o bem estar físico e a qualidade de vida entre os trabalhadores. Outra perspectiva seria aplicar a pesquisa em outras empresas, para se atingir um maior número de trabalhadores avaliados e se ter mais informações sobre essa população, subsidiando ações de vigilância em saúde do trabalhador.

\section{REFERÊNCIAS}

ASSOCIAÇÃO BRASILEIRA PARA O ESTUDO DA OBESIDADE E DA SÍNDROME METABÓLICA. Diretrizes brasileiras de obesidade 2016. 4 ed. São Paulo, 2016. Disponível em: <http://www.abeso.org.br/uploads/downloads/92/57fccc403e5da.pdf〉. Acesso em: 20 out. 2016

ANDERSON, Laurie M. et al. The Effectiveness of Worksite Nutrition and Physical Activity Interventions for Controlling Employee Overweight and Obesity. American Journal of Preventive Medicine, [s.1.], v. 37, n. 4, p.340-357, out. 2009.

APOVIAN, Caroline M.. Obesity: Definition, Comorbidities, Causes, and Burden. The American Journal Of Managed Care, [s.1], v. 22, n. 7, p.176-185, jun. 2016. Disponível em: < http://www.ajmc.com/journals/supplement/2016/impact-obesity-interventionsmanaged-care/ obesity-definition-comorbidities-causes-burden/P-1>. Acesso em: 14 out. 2016.

BAECKE, J.A.; BUREMA, J; FRIJTERS, J. A short questionnaire for the measurement of habitual physical activity in epidemiological studies. American Journal Of Clinical Nutrition, [s.1.], v. 36, n. 5, p.936-942, nov. 1982.

BARRETO, Sandhi Maria et al. Análise da Estratégia Global para Alimentação, Atividade Física e Saúde, da Organização Mundial da Saúde. Epidemiologia e Serviços da Saúde, [s.1.], v. 14, n. 1, p.41-68, mar. 2005.

BEZERRA, Ilana Nogueira et al. Consumo de alimentos fora do domicílio no Brasil. Revista de Saúde Pública, [s.1.], v. 47, supl. 1, p.200-211, ago. 2013.

BEZERRA, Ilana Nogueira; SICHIERI, Rosely. Eating out of home and obesity: a Brazilian nationwide survey. Public Health Nutrition, [s.1.], v. 12, n. 11, p.2037-2043, 12 maio 2009.

BRASIL. Ministério da Saúde. Resolução no 466 de 12 de dezembro de 2012. Aprova diretrizes e normas regulamentadoras de pesquisa envolvendo seres humanos. Diário Oficial da União. Poder executivo, Brasília: Ministério da Saúde, 2013. Seção 1, p.59.

BRASIL. Ministério da Saúde. Secretaria de Vigilância em Saúde, Secretaria de Gestão Estratégica e Participativa. Vigitel 2016: vigilância de fatores de risco e proteção para doenças crônicas por inquérito telefônico. Brasília: Ministério da Saúde; 2017. 
CASTRO, Maria Beatriz Trindade de; ANJOS, Luiz Antonio dos; LOURENÇO, Paulo Maurício. Padrão dietético e estado nutricional de operários de uma empresa metalúrgica do Rio de Janeiro, Brasil. Cadernos de Saúde Pública, [s.1.], v. 20, n. 4, p.926-934, ago. 2004.

CHURCH, Timothy S. et al. Trends over 5 Decades in U.S. Occupation-Related Physical Activity and Their Associations with Obesity. Plos One, [s.1.], v. 6, n. 5, p.e19657, maio 2011.

DUFFEY, Kiyah J et al. Differential associations of fast food and restaurant food consumption with 3-y change in body mass index: The Coronary Artery Risk Development in Young Adults Study. The American Journal of Clinical Nutrition, v. 85, n. 1, p.201-208, jan. 2007.

FAGGION, S.A., MONTEIRO, M. Aspectos nutricionais em trabalhadores de uma empresa de tecnologia da informação. In: MENDES, R.T., VILARTA, R., GUTIERREZ, G.L. Qualidade de Vida e Cultura Alimentar, Campinas, p.107-115, 2009.

FERNANDES, Ângela Cristina Puzzi; VAZ, Aline Bueno. Perfil do índice de massa corporal de trabalhadores de uma empresa de construção civil. J Health Sci Inst, [s.1], v. 30, n. 2, p.144-149, 2012.

GUH, Daphne P et al. The incidence of co-morbidities related to obesity and overweight: A systematic review and meta-analysis. BMC Public Health, [s.1.], v. 9. 25 mar. 2009. Disponível em: <https://www.ncbi.nlm.nih.gov/pmc/articles/PMC2667420/pdf/1471-2458-988.pdf>. Acesso em: 14 out. 2016.

HÖFELMANN, Dorotéia Aparecida; BLANK, Nelson. Excesso de peso entre trabalhadores de uma indústria: prevalência e fatores associados. Revista Brasileira de Epidemiologia, [s.1], v. 12, n. 4, p.657-670, 2009.

INSTITUTO BRASILEIRO DE GEOGRAFIA E ESTATÍSTICA. Pesquisa de Orçamentos Familiares 2008-2009: Antropometria e estado nutricional de crianças, adolescentes e adultos no Brasil. Rio de Janeiro, 2010. Disponível em: < http://biblioteca.ibge. gov.br/visualizacao/livros/liv45419.pdf>. Acesso em: 03 jun. 2016.

INSTITUTO BRASILEIRO DE GEOGRAFIA E ESTATÍSTICA. Percepção do estado de saúde, estilo de vida e doenças crônicas. Brasil, grandes regiões e unidades da federação. Rio de Janeiro, 2014. Disponível em: <ftp://ftp.ibge.gov.br/PNS/2013/pns2013. pdf>. Acesso em: 10 nov. 2016.

KUNERT, Camila Silva; MORAIS, Mariana Patrício de; CARVALHO, Ana Clara Martins e Silva. Salt and fat contents in preparations at commercial restaurants in Goiânia - GO.

Revista Brasileira em Promoção da Saúde, Fortaleza, v. 26, n. 1, p.18-25, jan./mar. 2013.

POPKIN, Barry M; ADAIR, Linda S; NG, Shu Wen. Global nutrition transition and the pandemic of obesity in developing countries. Nutrition Reviews, [s.1.], v. 70, n. 1, p.3-21, jan. 2012.

SANTOS, Melina Valério dos et al. Os restaurantes por peso no contexto de alimentação saudável fora de casa. Revista de Nutrição, Campinas, v. 24, n. 4, p.641-649, jul./ago. 2011.

SARNO, Flávio; BANDONI, Daniel Henrique; JAIME, Patrícia Constante. Excesso de peso e hipertensão arterial em trabalhadores de empresas beneficiadas pelo Programa de 
Alimentação do Trabalhador (PAT). Revista Brasileira de Epidemiologia, São Paulo, v. 11, n. 3, p.453-462, 2008.

SCHULTE, Paul A. et al. Work, Obesity, and Occupational Safety and Health. American Journal of Public Health, [s.1.], v. 97, n. 3, p.428-436, mar. 2007.

SEBASTIÃO, Helena Marina et al. Avaliação da qualidade de vida e do consumo alimentar de funcionários de uma empresa de fornecimento de energia elétrica. Revista Brasileira de Qualidade de Vida, [s.1.], v. 7, n. 1, p.12-19, 2 abr. 2015.

SIMON, Miriam Isabel Souza dos Santos et al. Avaliação nutricional dos profissionais do serviço de nutrição e dietética de um hospital terciário de Porto Alegre. Cadernos Saúde Coletiva, [s.1.], v. 22, n. 1, p.69-74, mar. 2014.

SOUZA, Amanda de M. et al. Alimentos mais consumidos no Brasil: Inquérito Nacional de Alimentação 2008-2009. Revista de Saúde Pública, [s.1.], v. 47, n. 1, p.190-199, fev. 2013.

STOLTE, Desire; HENNINGTON, Élida Azevedo; BERNARDES, Jefferson de Souza. Sentidos da alimentação e da saúde: contribuições para a análise do Programa de Alimentação do Trabalhador. Cadernos de Saúde Pública, [s.1.], v. 22, n. 9, p.1915-1924, set. 2006.

TABAK, Rachel G. et al. Workplace Social and Organizational Environments and HealthyWeight Behaviors. Plos One, [s.1.], v. 10, n. 4, p. e0125424, 28 abr. 2015. Disponível em: <http://journals.plos.org/plosone/article?id=10.1371/journal.pone.0125424>. Acesso em: 19 out. 2016.

WORLD HEALTH ORGANIZATION. Diet, nutrition, and the prevention of chronic diseases. World Health Organization technical report series, Geneva, v. 916, n.5, p.1-149, 2003.

WORLD HEALTH ORGANIZATION. Physical status: the use and interpretation of anthropometry. World Health Organization technical report series, Geneva, v. 854, p.1452, 1995. 